\title{
Fatiga y calidad de vida en niños, niñas y adolescentes con cáncer hematológico
}

\begin{abstract}
*Correspondencia:
\end{abstract}
javierdx@hotmail.es

Av. Carlos Julio Arosemena Km 1 $1 / 2$, Guayaquil - Ecuador. Teléfono: (593) [04] 3804600

Conflicto de intereses: LoS autores declaran no tener conflictos de intereses

Recibido: 2 de Febrero 2021 Aceptado: 20 de Marzo, 2021 Publicado: 30 de Abril, 2021 Editor: Dr. Felipe Campoverde

\section{Membrete bibliográfico:} Espín J, Camacho E, Jurado S, Sánchez R, Sierra V, Abril T, Méndez B, Noblecilla J. Fatiga y calidad de vida en niños, niñas y adolescentes con cáncer hematológico. Rev. Oncol. Ecu 2021;31(1):56-65.

ISSN: $2661-6653$

DOI: https://doi.org/10.33821/548

Copyright Espín J, et al. Este artículo es distribuido bajo los términos de Creative Commons Attribution License, el cual permite el uso y redistribución citando la fuente $y$ al autor original.

\section{Fatigue and quality of life in children and adolescents with hematological cancer}

Javier Andrés Espín Jordán*1, Erick Andrés Camacho Rodriguez1, Stalin Augusto Jurado Auria1 ${ }^{(D)}$, Rubén Eduardo Sánchez Astudillo1, Víctor Hugo Sierra Nieto1 ${ }^{(D)}$, Tania María Abril Mera1 ${ }^{(D)}$, Boris José Méndez Pérez1D, Joselyne Angélica Noblecilla Troya1 ${ }^{1 D}$.

1. Carrera de Terapia Física, Facultad de Ciencias Médicas, Universidad Católica Santiago de Guayaquil.

\section{Resumen}

Introducción: La fatiga es un problema relevante en pacientes con cáncer hematológico y puede ser un motivo de reducción importante de la calidad de vida. El objetivo del presente estudio fue realizar un estudio descriptivo de un grupo de niños y adolescentes portadores de neoplasias de origen hematológico en un centro único de referencia.

Metodología: El presente estudio observacional tiene un corte retrospectivo, en donde se incluyeron pacientes menores a 18 años con el diagnóstico de cáncer hematológico en quienes se revisó la historia clínica y se realizó un test de calidad de vida. Se realiza un análisis de correlación entre las variables usando estadística no paramétrica.

Resultados: ingresaron al estudio 64 pacientes. De sexo masculino ( $n=37)$ y femenino $(n=27)$. El valor medio de fatiga fue ( $5.72 \pm 0.53$ y $5.85 \pm 0.51$ ), en actividad física y salud ( $1.49 \pm 0.17$ y $1.44 \pm 0.13$ ) en hombres y mujeres respectivamente. El valor medio de edad fue de $5.81 \pm 2.46$ en el rango de 2 a 10 años y $14.55 \pm 2.15$ en el rango de 11 a 18 años. Según el rango etario existió más predominancia de fatiga en niños que en adolescentes y más en mujeres que en hombres.

Conclusión: No se encontró relación entre las variables de estudio. Sin embargo, los hallazgos expuestos indican una marcada sensación de fatiga siendo esta moderada pudiendo convertirse en severa; de igual manera se obtuvo niveles bajos y medios de calidad de vida en las similares dimensiones incluidas en las baterías de evaluación; sugiriendo futuras investigaciones incluyendo colaboración de abordaje multidisciplinaria para llegar a impartir las terapias adecuadas que beneficien a los pacientes con cáncer.

Palabras claves:

DeCS: Neoplasias hematológicas, Fatiga, Epidemiología, Investigación sobre Servicios de Salud, Calidad de vida, niño, adolescente, Encuestas y cuestionarios.

DOI: $10.33821 / 548$ 


\begin{abstract}
Introduction: Fatigue is a relevant problem in patients with hematological cancer and can be a reason for a significant reduction in quality of life. The objective of this study was a descriptive study of a group of children and adolescents with neoplasms of hematological origin in a single referral center.

Methodology: The present observational study has a retrospective cut-off, which included patients under 18 years of age with a diagnosis of hematological cancer in whom the clinical history was reviewed and a quality of life test was performed. A correlation analysis between the variables is performed using nonparametric statistics.
\end{abstract}

Results: 64 patients entered the study. Male $(n=37)$ and female $(n=27)$. The mean value of fatigue was (5.72 \pm 0.53 and $5.85 \pm 0.51$ ), in physical activity and health $(1.49 \pm 0.17$ and $1.44 \pm 0.13$ ) in men and women respectively. The mean age value was $5.81 \pm 2.46$ in the range of 2 to 10 years and $14.55 \pm 2.15$ in the range of 11 to 18 years. According to the age range, there was more prevalence of fatigue in children than in adolescents and more in women than in men.

Conclusion: No relationship was found between the study variables. However, the results shown indicate a marked feeling of fatigue, this being moderate and it can become severe; in the same way, low and medium levels of quality of life were obtained in the similar dimensions included in the evaluation batteries; suggesting future research including collaboration of a multidisciplinary approach to arrive at the delivery of appropriate therapies that benefit cancer patients.

\title{
Keywords:
}

MESH: Hematologic Neoplasms; Fatigue; Epidemiology, Health Services Research; Quality of life; Child, adolescent; Surveys and Questionnaire.

DOI: $10.33821 / 548$

\section{Introducción}

La fatiga es el síntoma más común que experimentan los pacientes durante la trayectoria del cáncer desde el diagnóstico hasta la final de la vida y se define como una sensación angustiosa, persistente y subjetiva de cansancio o agotamiento físico, emocional y / o cognitivo relacionado con el cáncer o el tratamiento del cáncer que no es proporcional a la actividad física reciente y que interfiere con el funcionamiento habitual [1].

Se conoce que la fatiga tiende a mejorar con el tiempo después del tratamiento oncológico, pero puede dar mayores síntomas de depresión y menor movilidad en sobrevivientes de cáncer infantil [2].

No existe correlación entre el nivel de fatiga, la edad o el sexo. En un estudio de adolescentes con púrpura trombocitopénica idiopática (PTI) persistente y recién diagnosticada tenían puntuaciones medias de fatiga más altas que aquellos con PTI crónica $(P=0.03)$. La fatiga mejoró significativamente en niños y adolescentes 1 mes después de comenzar los tratamientos de 
segunda línea, y esta mejoría continuó presente a los 12 meses después de comenzar el tratamiento. Las puntuaciones de fatiga en todos los puntos de tiempo se correlacionaron con la calidad de vida general utilizando la herramienta Kids ITP, pero no se correlacionaron con los síntomas de hemorragia, el recuento de plaquetas o la respuesta de las plaquetas al tratamiento [3].

Con estos antecedentes se planteó la pregunta de investigación si la fatiga disminuye la calidad de vida en niños con cáncer de origen hematológico por lo realizamos el presente estudio observacional para resolver esta pregunta.

\section{Materiales y métodos \\ Diseño del estudio}

El presente estudio es observacional, descriptivo, transversal.

\section{Área de estudio}

El estudio se realizó en el servicio de hospitalización del Instituto Oncológico Nacional "Dr. Juan Tanca Marengo" de la Sociedad de Lucha contra el Cáncer-SOLCA, Guayaquil Ecuador, el período estudio fue del 1ro de enero del 2019 al 31 de diciembre del 2019.

\section{Universo y muestra}

El universo fue conformado por todos los pacientes hospitalizados en la institución de edades comprendidas de 2 a 18 años con diagnóstico de cualquier tipo de leucemias. Se utilizó para la identificación el código internacional de enfermedades CIE-10, con los códigos desde C1 hasta C95. El cálculo del tamaño muestral fue no probabilístico, tipo censo en donde se incluyeron todos los casos posibles de la institución en las fechas establecidas.

\section{Participantes}

Se incluyeron casos en quienes se registraron el ingreso por primera ocasión a la institución con el diagnóstico de Leucemia con edades comprendidas entre los 0 años hasta la edad máxima registrada en la base de datos. Se excluyeron casos en quienes no se completó las variables estudiadas.

\section{Variables}

Las variables fueron diagnóstico, edad, sexo, calidad de vida (Formulario Kidscreen 52), fatiga (Formulario IBF).

\section{Procedimientos, técnicas e instrumentos.}

Los datos fueron recogidos de la historia clínica en un formulario diseñado exclusivamente para tal propósito. Para garantizar la confiabilidad de la información los investigadores fueron entrenados sobre la recolección de los datos. Se utilizó el Inventario Breve de la Fatiga el cual se basó en los criterios de los factores de la actividad en general, estado de ánimo, capacidad para caminar, trabajo normal (ya sea en casa o fuera del hogar), relaciones con otras personas, capacidad de diversión (disfrutar la vida), consistía en encerrar con un círculo un número de una escala numérica que comienza desde 0 cuyo significado es que no infiere y llega hasta 
10 cuyo significado es que infiere por completo, de la manera en que la fatiga ha interferido durante las últimas 24 horas, categorizando las puntuaciones en donde 0 significa: "sin fatiga"; 1-3 "leve"; 4-6 "moderada" y 7-10: "severa"(12), también se midió en estos pacientes la calidad de vida con el test Kidscreen52 modificado en el cual pedía que se anotara la fecha actual de realización, sexo, fecha de nacimiento, información personal y si tenía algún antecedente patológico personal para los resultados se basó en los criterios actividad física y salud, estado de ánimo, vida familiar, asuntos económicos (padres), tus amigos/as y en el colegio, que incluían preguntas en cada criterio, donde se da a escoger 5 opciones que son: "nada" cuyo porcentaje va de 0-20; "un poco" 21-40, "moderadamente" 41-60, "mucho" 61-80, "muchísimo" 81-100, las puntuaciones directas de las dimensiones se obtienen de la media de los 5 ítems, los resultados se convierten a un rango de 0 a 100 puntos y con este puntaje se determina alta o baja calidad de vida (13)

\section{Análisis estadístico}

Recopilada la información se ingresó en una matriz de datos del software SPSS ${ }^{\text {TM }} 24.0$ (IBM, Armonk, NY, EEUU). Se utilizó estadística descriptiva en base a frecuencias y porcentajes para las variables cualitativas y para las cuantitativas medidas de tendencia central. Se realizó estadísticas descriptivas sobre las variables de estudio, en donde se calculó el valor promedio de fatiga y de calidad de vida calculándolas por dimensiones y desviación estándar. Se utilizó la prueba no paramétrica U-Mann Whitney para la comparación de grupos, tanto para el sexo como para la edad, y se utilizó la prueba de coeficiente de correlación de Spearman para relación de las variables.

\section{Resultados}

El estudio incluyó 64 pacientes.

\section{Caracterización demográfica}

La tabla 1 presenta la media de las variables fatiga y calidad de vida según el sexo, compuesta por una muestra de 64 pacientes con cáncer hematológico hospitalizados, de los cuales 37 son sexo masculino y 27 son de sexo femenino. El valor medio de fatiga es de $5.72 \pm 0.53$ y $5.85 \pm 0.51$ en hombres y mujeres respectivamente. En la actividad física y salud el valor medio fue de $1.49 \pm 0.17$ en hombres y $1.44 \pm 0.13$ en mujeres. Por su parte el estado de ánimo presenta un valor medio en hombres de $3.59 \pm 0.35$ y en mujeres $3.43 \pm 0.40$.

La tabla 2 muestra la comparación de las variables fatiga y calidad de vida según la edad, integrada por los rangos de 2 a 10 años para los niños y niñas con un valor medio de $5.81 \pm$ 2.46 , y de 11 a 18 años una media de $14.55 \pm 2.15$. Como podemos ver al igual que en la tabla 1 no hay una diferencia significativa entre los 2 grupos.

La tabla 3 presenta indica la baja o ausencia relación entre la fatiga con las dimensiones de la calidad de vida, lo que significa que las variables son independientes, existe evidencia de no correlación entre las variables. 
En la figura 1 se muestra mayor predominancia de fatiga en niños que en adolescentes, por encima de los valores medios de categoría moderada, acercándose a convertirse en fatiga severa.

En el figura 2 se representan valores elevados de fatiga por encima de la media, para la categoría moderada en ambos sexos, como se mencionó en el gráfico 1, los valores pueden llegar a aumentar al diagnóstico de fatiga severa.

Tabla 1 Fatiga y calidad de vida según el sexo en niños, niñas y adolescentes con cáncer hematológico.

\begin{tabular}{lccc}
\hline \multicolumn{1}{c}{ Variable } & Sexo masculino $\mathrm{n}=37$ & Sexo Femenino $\mathrm{n}=27$ & $P$ \\
\hline Edad & $8.76 \pm 2.46$ & $8.89 \pm 4.99$ & 0.962 \\
IBF & $5.72 \pm 0.53$ & $5.85 \pm 0.51$ & 0.407 \\
CDV: Actividad física y salud & $1.49 \pm 0.17$ & $1.44 \pm 0.13$ & 0.275 \\
CDV: Estado de ánimo & $3.59 \pm 0.35$ & $3.43 \pm 0.40$ & 0.076 \\
CDV: Vida familiar & $4.30 \pm 0.01$ & $4.30 \pm 0.01$ & 1 \\
CDV: Asuntos económicos & $3.00 \pm 0.01$ & $3.00 \pm 0.01$ & 1 \\
CDV: Amistades & $2.91 \pm 0.23$ & $2.89 \pm 0.23$ & 0.938 \\
CDV: Colegio & $3.99 \pm 0.13$ & $3.98 \pm 0.14$ & 0.589
\end{tabular}

IBF: Inventario breve de fatiga. CDV: Calidad de Vida.

Tabla 2 Fatiga y calidad de vida según la edad en niños, niñas y adolescentes con cáncer hematológico

\begin{tabular}{lccc}
\hline \multicolumn{1}{c}{ Variable } & Pacientes de 2 a 10 años $n=42$ & Pacientes de 11 a 18 años $n=22$ & $P$ \\
\hline Edad & $5.81 \pm 2.46$ & $14.55 \pm 2.15$ & $<0.0001$ \\
IBF & $5.90 \pm 0.52$ & $5.55 \pm 0.46$ & 0.003 \\
CDV: Actividad física y salud & $1.46 \pm 0.14$ & $1.49 \pm 0.17$ & 0.399 \\
CDV: Estado de ánimo & $3.52 \pm 0.41$ & $3.53 \pm 0.33$ & 0.824 \\
CDV: Vida familiar & $4.30 \pm 0.01$ & $4.30 \pm 0.01$ & 1 \\
CDV: Asuntos económicos & $3.00 \pm 0.01$ & $3.00 \pm 0.01$ & 1 \\
CDV: Amistades & $2.88 \pm 0.23$ & $2.93 \pm 0.232$ & 0.332 \\
CDV: Colegio & $3.97 \pm 0.14$ & $4.00 \pm 0.14$ & 0.463 \\
\hline
\end{tabular}

IBF: Inventario breve de fatiga. CDV: Calidad de Vida.

Tabla 3 Relación entre fatiga y calidad de vida en niños, niñas y adolescentes con cáncer hematológico.

\begin{tabular}{lcc}
\hline & Coeficiente de correlación de Spearman & $P$ \\
\hline IBF \& CDV: Actividad física y salud & 0.102 & 0.424 \\
IBF \& CDV: Estado de ánimo & 0.102 & 0.422 \\
IBF \& CDV: Amistades & -0.204 & 0.106 \\
IBF \& CDV: Colegio & -0.146 & 0.251 \\
\hline
\end{tabular}




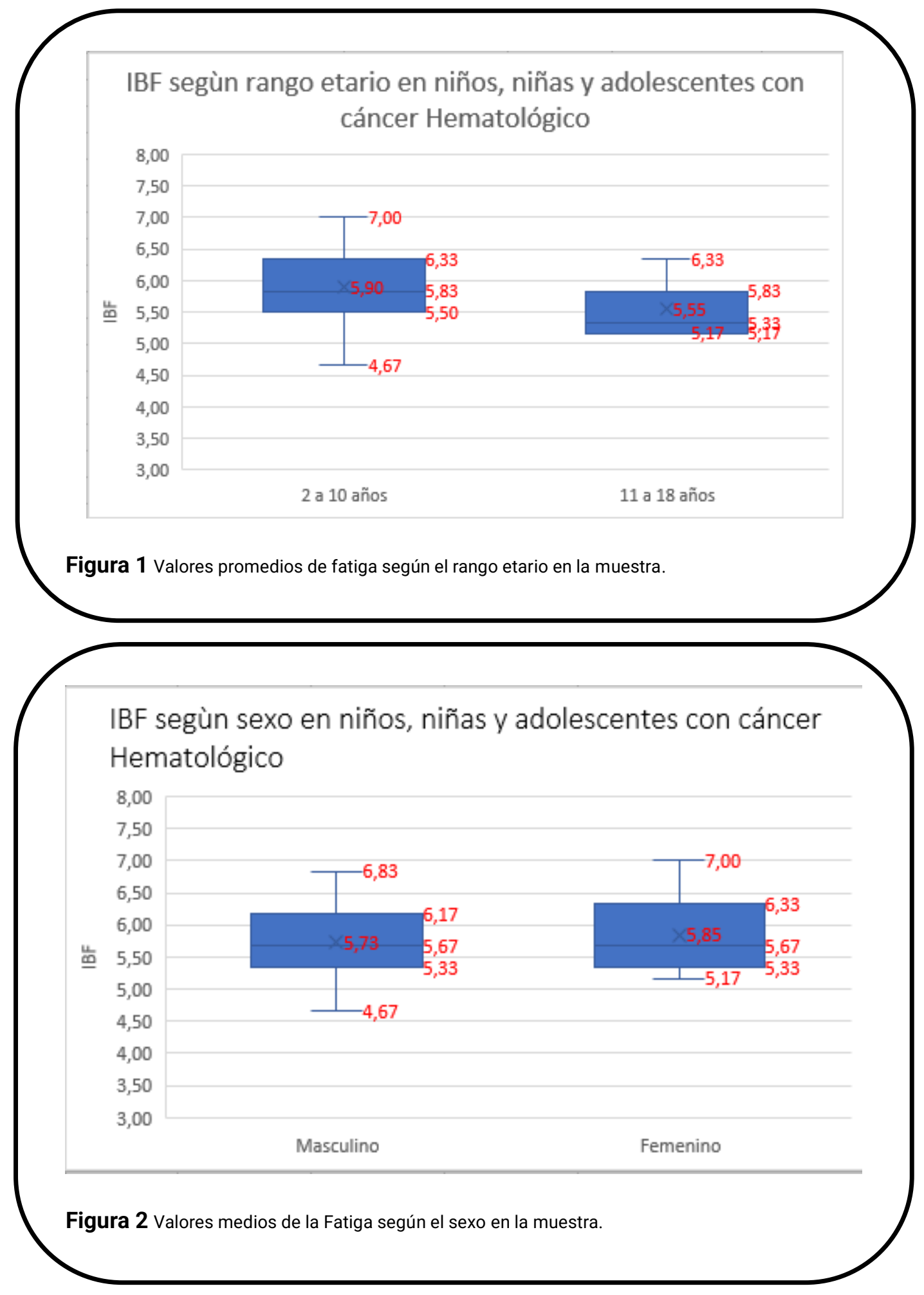




\section{Discusión}

Como resultado los datos indican que no existe una relación positiva entre la variable fatiga con la calidad de vida relacionada a la salud en ningunas de sus dimensiones en niños, niñas y adolescentes con cáncer hematológico en el presente estudio, sin embargo un estudio realizado en la Barcelona, España, indica que hay una relación muy alta entre ambas variables [14]. Debido a que la fatiga se convierte en un síntoma irritante, superando las sensaciones como el dolor, vértigo y náuseas, incluso este síntoma se conserva aún después de que el paciente de por finalizado el tratamiento [15], como consecuencia del cáncer o terapia y no por resultado de la actividad física conocido como el síndrome de fatiga relativa afectando del $70 \%$ al $96 \%$ de los pacientes [16]. Se conoce que los cambios físicos y comportamentales producto del cáncer llega a afectar alguna de las dimensiones asociadas a la salud, y repercutirá directamente en la disminución de la calidad de vida del paciente [17]. En los niños, este problema se presenta como una sensación física, y en los adolescentes en elementos emocionales, físicos y mentales, evidenciando que se convierte en un factor estresante en el desarrollo de la calidad de vida en los niños y adolescentes con esta patología [18].

Las limitaciones del presente estudio comprenden la dificultad del diseño transversal para analizar la causa y efecto y que el muestreo no probabilístico permite dar resultados solo en la población evaluada. La información de las preguntas (físicas, psicológicas, económicas y sociales) percibidas por los pacientes podría incitar respuestas incorrectas, debido a que los niños comprenden de manera diferente de acuerdo a su edad e influencia o interpretación por parte de los padres. Además de que la muestra del estudio es pequeña de 64 pacientes con cáncer hematológico hospitalizados, dio la predisposición que exista mucha dispersión sin dar una tendencia lineal en los resultados que no sean significativos lo que ocurre solo en un resultado. Entre las fortalezas de la investigación está la inclusión de la relación de las dos variables de estudios al grupo poblacional dirigido a niños, niñas y adolescentes en donde se dio como resultado la existencia de fatiga moderada en las diversas actividades en su vida cotidiana llegando a poder convertirse en severa [19].

Según un estudio realizado en Bogotá, Colombia, en el mundo la tasa de incidencia del cáncer infantil es de 50-200 por cada millón de niños [20]. El cáncer hematológico entre todos los tipos de cáncer representa el $25 \%$ en niños y adolescentes siendo así la más frecuente [16]. Se sabe que al niño diagnosticado con cáncer, presenta un sin número de diferentes emociones: temor, enojo, nostalgia, desanimo o depresión perjudicando sus actividades en su entorno escolar y familiar [21]. Por lo tanto, la calidad de vida relaciona a la salud solo la puede percibir el paciente sobre el impacto de su bienestar y funcionalidad [22].

En la actualidad existen pocos estudios correlacionales entre la fatiga y la calidad de vida, poniendo en manifiesto futuras investigaciones para clarificar los efectos favorables o desfavorables al aumentar o disminuir alguna de las dos variables entre sí en los pacientes con cáncer; Como se describe en un estudio realizado en el estado de São Paulo, Brasil reciente del año 2019, la fatiga relacionada al cáncer tiene mayor intensidad y más duración que la fatiga típica afectando en las decisiones terapéuticas en el curso del tratamiento [23]. Por lo tanto, afecta la capacidad funcional al momento de realizar actividades diarias, además de la 
Nota del Editor La Revista Oncología Ecu permanece neutral con respecto a los reclamos jurisdiccionales en mapas publicados y afiliaciones institucionales. disminución de la motivación, poca energía y sufrimiento [24]. Recientemente los índices de supervivencia de los pacientes pediátricos con cáncer hacen esencial el abordaje de nuevos problemas clínicos [25].

\section{Conclusiones}

No se encontró relación entre las variables de estudio. Sin embargo, los hallazgos expuestos indican una marcada sensación de fatiga siendo esta moderada pudiendo convertirse en severa; de igual manera se obtuvo niveles bajos y medios de calidad de vida en las similares dimensiones incluidas en las baterías de evaluación; sugiriendo futuras investigaciones incluyendo colaboración de abordaje multidisciplinaria para llegar a impartir las terapias adecuadas que beneficien a los pacientes con cáncer.

\section{Agradecimientos}

Los autores agradecen a todas las personas de la Institución que colaboraron en el desarrollo de esta investigación.

\section{Información administrativa}

Abreviaturas

IBF: Inventario breve de fatiga. CDV: Calidad de vida.

Archivos Adicionales

Ninguno declarado por los autores.

Fondos

Los autores no recibieron ningún tipo de reconocimiento económico por este trabajo de investigación.

Disponibilidad de datos y materiales

Existe la disponibilidad de datos bajo solicitud al autor de correspondencia. No se reportan otros materiales.

Contribuciones de los autores 
Todos los autores realizaron por igual la conceptualización, curación de datos, análisis formal, adquisición de fondos, investigación, recursos, software, redacción - borrador original, supervisión, validación, visualización, metodología, administración de proyecto, escritura: revisión y edición.

Todos los autores leyeron y aprobaron la versión final del manuscrito.

Aprobación del comité de ética

El protocolo de investigación fue aprobado por el Departamento de Docencia del Hospital Solca - Guayaquil y las autoridades institucionales y por el comité de bioética de la Facultad de Ciencias Médicas de la Universidad Católica Santiago de Guayaquil.

Consentimiento para publicación

El presente estudio es un análisis de base de datos, no aplica para este tipo de estudio.

\section{Referencias}

1. Fabi A, Bhargava R, Fatigoni S, Guglielmo M, Horneber M, Roila F, Weis J, Jordan K, Ripamonti Cl; ESMO Guidelines Committee. Electronic address: clinicalguidelines@esmo.org. Cancer-related fatigue: ESMO Clinical Practice Guidelines for diagnosis and treatment. Ann Oncol. 2020 Jun;31(6):713-723. doi: 10.1016/j.annonc.2020.02.016. Epub 2020 Mar 12. PMID: $\underline{32173483}$.

2. Karimi M, Cox AD, White SV, Karlson CW. Fatigue, Physical and Functional Mobility, and Obesity in Pediatric Cancer Survivors. Cancer Nurs. 2020 Jul/Aug;43(4):E239-E245. doi: 10.1097/NCC.0000000000000712. PMID: 30925511.

3. Grace RF, Klaassen RJ, Shimano KA, Lambert MP, Grimes A, Bussel JB, Breakey VR, Pastore YD, Black V, Overholt K, Bhat R, Forbes PW, Neunert C. Fatigue in children and adolescents with immune thrombocytopenia. Br J Haematol. 2020 Oct;191(1):98-106. doi: 10.1111/bjh.16751. Epub 2020 Jun 5. PMID: 32501532.

4. Buscemi V, Font A, Rodríguez E. Fatiga, expectativas y calidad de vida en cáncer. Psicooncología. 2004;1:45-56. SU: ucm.es/PSIC0404120045A

5. Arguedas CV, Villalobos VU, Cerdas JPG, Argüello ABA. Síndrome de fatiga relacionado con cáncer. Revista Clínicade la Escuela de Medicina UCR -HSJD 2015;5(4):32-28. doi: 10.15517/RC_UCR-HSJD.V5I4.20774

6. Reyes MCR, Canchila DMG. Efectos de la quimioterapia en el sistema musculoesquelético de niños y adolescentes con leucemia linfoblástica aguda. UNIVERSIDAD Y SALUD. 2014;16:8.

7. Cabezas Quiroga JS, Madrid Castro LP. Percepción de la calidad de vida en niños y adolescentes con cáncer en Villavicencio Meta. Universidad Cooperativa de Colombia Villavicencio Meta. 2017;

8. Ramírez JS, Carrillo GM. Evaluación de síntomas en niños y adolescentes con cáncer: revisión integrativa.: Evaluation of symptoms in children and adolescents with cancer: comprehensive review. Revista Ciencia y Cuidado. 2019;16(3):103-13.

9. Leon M. Protección social de la niñez en el Ecuador [Internet]. CEPAL/ UNICEF; 2017 [cited 2020 Aug 24]. Available from: https://www.cepal.org/es/publicaciones/41233-proteccion-social-la-ninez-ecuador

10. OMS. Datos y cifras sobre el cáncer [Internet]. WHO. World Health Organization; [cited 2020 May 21]. Available from: https://www.who.int/cancer/about/facts/es/ 
11. Blandón MMM, Erazo MJV, Guarín SR. EXPERIENCIAS DE NIÑOS CON LEUCEMIA, PERTENECIENTES A LA FUNDACIÓN “NIÑO LEUCÉMICO” DE LA CIUDAD DE POPAYÁN. 2013. 7:14.

12. Gordillo Altamirano F, Fierro Torres MJ, Cevallos Salas N, Cervantes Vélez MC. La salud mental determina la calidad de vida de los pacientes con dolor neuropático oncológico en Quito, Ecuador. RCP. 2017 Jul 1;46(3):154-60.

13. Avelar JM de P, Nicolussi AC, Toneti BF, Sonobe HM, Sawada NO, Avelar JM de P, et al. Fatigue in patients with head and neck cancer undergoing radiation therapy: a prospective study. Revista Latino-Americana de Enfermagem [Internet]. 2019 [cited 2020 Aug 24];27. Available from:

http://www.scielo.br/scielo.php?script=sci_abstract\&pid=S0104-11692019000100352\&lng=en\&nrm=iso\&tlng=es

14. Silva MCM da, Lopes Júnior LC, Nascimento LC, Lima RAG de. Fatigue in children and adolescents with cancer from the perspective of health professionals. Rev Latino-Am Enfermagem [Internet]. 2016 [cited 2020 Jun 29];24(0). Available from: http://www.scielo.br/scielo.php?script=sci_arttext\&pid=S0104-

$11692016000100405 \& \operatorname{lng}=e n \&$ tlng=en

15. Plasencia Lugo LI, Campo Díaz M, Coro Carrasco Z. La reincorporación social y calidad de vida en niños con leucemia. Revista de Ciencias Médicas de Pinar del Río. 2017 Aug;21(4):14-23. 